\title{
A Gravitational Search Algorithm for effective Web Service Selection for Composition with enhanced QoS in SOA
}

\author{
D.Palanikkumar \\ Asst Professor \\ Department of CSE \\ Anna University of Technology, \\ Coimbatore
}

\author{
P.Anbuselvan \\ PG Scholar \\ Department of CSE \\ Anna University of Technology, \\ Coimbatore
}

\author{
B.Rithu \\ PG Scholar \\ Department of CSE \\ Anna University of Technology, \\ Coimbatore
}

\begin{abstract}
Web services selection and composition is a new software development paradigm to achieve service oriented computing. QoS of compound service is a key factor for satisfying the users' needs. The services selection algorithm based on quality of services is a combinatorial optimization problem. For Web services selection for composition based on QoS, the optimization algorithms can solve it well. Genetic Algorithm and Gravitational Search Algorithm are proposed to solve this issue. In order to meet the QoS requirements of consumers, this paper presents the QoS calculation of non functional requirements such as cost, availability, reliability and execution time. To verify the effectiveness in latency of Web Services selection the above two algorithms are compared. Results indicate the Gravitational Search Algorithm improves the latency over the Genetic algorithm.
\end{abstract}

Key words: GA, GSA, Service Selection.

\section{INTRODUCTION}

Web service is a technology that provides flexibility and interconnection between different distributed applications over the Internet and intranets. Web service composition is gaining a considerable momentum as an approach to the effective integration of distributed, heterogeneous, and autonomous application[20].The process is to search for the optimal set of services that can be composed to create a new service, result in the best QoS with user constraints $[15,18]$. Web service has three basic underlying components which form its basic platform; WSDL, UDDI and SOAP $[16,20]$. These components are used to select the suitable web services based on client's request. WSDL (Web Service Description Language) is an XML based language. It is used to describe the web services with its functionalities and provides an end point to invoke the web service. A web service is described in the standard XML format of service description using WSDL. It has similar purpose of IDLs (Interface Definition Language) [8, 14]. UDDI have enabled service providers and requesters to find the web service through UDDI Business Registries $[17,23]$. In order to select a suitable web services that are published and made available to the clients, every web service must initially publish in some UDDI registries [11, 14].SOAP defines how to communicate the information using XML in web service selection. SOAP specifies the message format and description of message should be transported using HTTP and SMTP [9, 14].The basic web service model consists of three entities: Service Provider, Service Registry and Service Requester [20]. Service Provider is used to provide the service to the Service Registry [14]. Service Requester is the requester who retrieves the information from service registry to find a suitable service provider and publish the web service. The Service Registry contains the information about Service Provider and Service Requester [10].Genetic algorithm is an approximate algorithm and used to solve optimization combinatorial problem. With the rapid development of web service technology, many service providers have same functionalities and different QoS [12]. Gravitational Search Algorithm is used in an NP hard problem area in a large power system. This optimization deals to find the best location of SVC (Static Var Compensator). The optimization is made on two parameters: location and size [7]. The problem of web service selection for composition can be defined as follows, "In an algorithm based approach of web service selection, which evolutionary search and optimization technique can obtain the best result". This paper presents a comparative approach to Service selection for Service Composition based on QoS with the user's constraints. Genetic Algorithm is the most conventional method of problem solving involving problems with more solution space. Gravitational Search Algorithm (GSA) is one of the optimization algorithms based on law of gravity $[1,5]$.

\section{RELATED WORK}

\subsection{Web Services selection based on QoS}

\section{Measures}

Many works has been carried out by different researchers for web service selection based on QoS for end-to-end composition. In the web service more service properties are considered in the service selection, especially nonfunctional service properties, known as Quality of services $[15,19]$. QoS is a set of parameters that comprise of 
response time, price, reputation, correctness etc [19] Works had been done to apply Collaborative filtering (CF) in web service recommendation [4]. The QoS based service selection executes on two approaches, one is based on local optimization and other is global planning [18]. The aim of the study is for the optimal set of services, composed to create a new service and best QoS under the service constraints. As the result, the web services composition is done by combining local strategy and global planning for QoS constraints [3, 18].

Fig.1 indicates the path selection of web service and search the optimal path of the best service in the n number of $(\mathrm{G} 1$, $\mathrm{G} 2 \ldots, \mathrm{Gn})$ services in the user's needs.

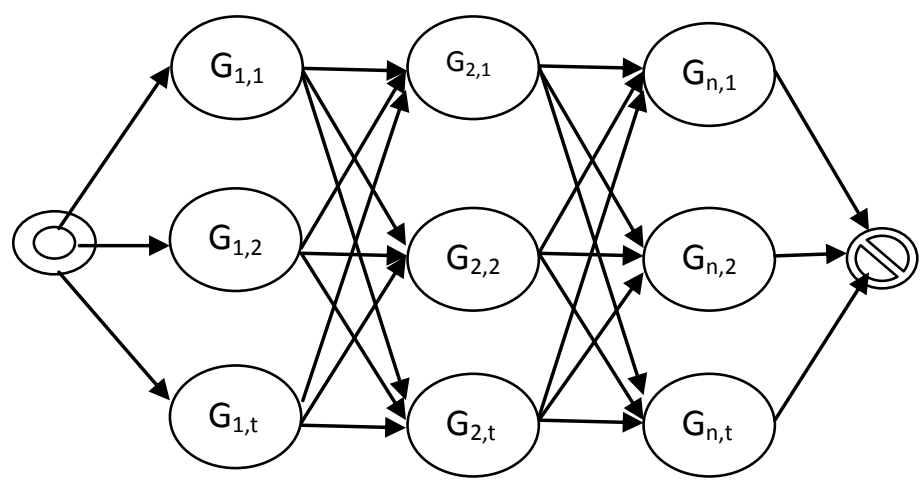

Fig .1 Web Service Composition based on QoS

The Web Service Composition provides a value-added service such as integration and interaction of the existing component services [2]. The Service Composition becomes a decision problem on which component services should be selected the user's functional [21] and nonfunctional [22] requirements. Web services have important implication for business-to-business transaction [9]. In order to satisfy the multiple requirements, suitable component services need to be selected for service composition process. The Selection of these web services is an important part of web services composition. Internet provides options to the user's to cater their needs by finding exact web service $[2,8]$. Since the web services composition based on QoS is a NP hard problem, the bionics optimization algorithms can solve it well. On the other hand, QoS of compound service is a key factor for satisfying the user's needs [1]. The users prefer different QoSs according to their desires [21]. The services Selection algorithm based on quality of services by gravitational search algorithm (GSA), which is one of the recent optimization algorithms [5]. GSA has many merits, like rapid convergence speed, less memory use, considering a lot of special parameters such as the distance between solutions, etc.

\subsection{The web services selection problem}

Motivating Example: In this section, we present a travel plan domain such as hotel, airline and taxi reservation web service that receives information regarding the type of customer wishes to reserve and makes a reservation to customer needs. Fig. 2 has shown the diagram for this model. This study describes how Genetic Algorithm and Gravitational Search Algorithm can be applied to the optimization problem of optimal web service selection and compares the performance of both the algorithms.

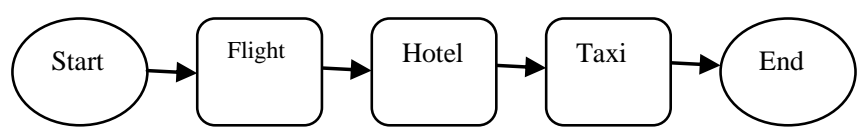

Fig.2 Travel plan domain

\section{WEB SERVICE SELECTION BASED ON GENETIC ALGORITHM}

Genetic algorithm (GA) [13] is an efficient Search method based on principles of Population genetic.GA is a tool to solve complex combinatorial optimization problems. It is based on the mechanism of evolution and solving intractable optimization Problem. This paper provides the optimal set of services, select the best QoS under the user constraints.

\subsection{Definition of Population}

Genetic Algorithm work with set of genomes called as population. Each genome represents the Web service selection process. This process generates several iteration process is for genetic operation. Each genome associated with the fitness value based on the fitness function.

\subsubsection{Genetic Operation}

Once genomes are defined, we need to reproduce the population by performing genetic operation such as Selection, Crossover and mutation.

Selection: This Process selects chromosomes in the population for reproduction.

Crossover: This Process randomly chooses a locus and exchanges the subsequences before and after that locus between two chromosomes to create two offspring.

Mutation: This Process randomly flips some of the bits in the chromosomes.

\subsubsection{GA for optimal service selection}

Step 1: Initialization.

Step 2: Evaluate the population.

Step 3: Genomes selection.

Step 4: Fitness value computation.

Step 5: Selection, Crossover and Mutation operation of genomes.

Step 6: Repeat step 3-5 until the cycle termination.

\section{WEB SERVICE SELECTION BASED ON GRAVITATIONAL SEARCH ALGORITHM}

Gravitational Search Algorithm is based on the law of gravity. It Consider agents as objects and their performance measured by their masses [24].

Now, consider the travel plan domain we which created a web service application for flight, hotel and taxi Reservation. The application uses non functional requirements such as price, duration, reputation, rate and availability. Each services have four candidate services.

The fitness value of each service is calculated in web application using the non functional parameter and stored into the new table.

Gravitational and inertial are calculated by the fitness evaluation. The gravitational and inertial masses are updating by the following equations:

$\mathrm{Mg}_{\mathrm{i}}=(f i t(t)-\operatorname{Worst}(t)) /(\operatorname{Best}(t)-\operatorname{Worst}(t))$ 
Here fit $(\mathrm{t})$ calculated by the non functional requirement parameters and represents the fitness value of the each services at time $\mathrm{t}$.

fit $(\mathrm{t})=\frac{\text { price }+ \text { duration }+ \text { reputation }+ \text { rate }+ \text { availability }}{\text { time }}$

Where fit $(t)$ represent the fitness value of the agents $i$ at time $t$, worst $(t)$ and best $(t)$ are defined as follows:

Best $(\mathrm{t})=$ Max fit $(\mathrm{t})$

Worst $(\mathrm{t})=$ Min fit $(\mathrm{t})$

At a specific time ' $\mathrm{t}$ ', the fitness value is calculated by:

$\mathrm{Fij}=\frac{\left(M g_{i} * g t\right)}{\left(R_{i j}(t)+e s\right)} * f i t(t)$

Here gt is gravitational constant at time t, es is small constant. fitness is fit $(\mathrm{t})$ value of each services.

$\mathrm{R}_{\mathrm{ij}}(\mathrm{t})=(\text { euclidian } 1+\text { euclidian } 2)^{2}$

$\mathrm{R}_{\mathrm{ij}}(t)$ is the Euclidian distance between two services. Here we can calculate the Euclidian1 using the flight and hotel services and Euclidian2 using the Euclidian1 and car services.

Euclidian1 $=(\text { flight service }- \text { hotel service })^{2}$

Euclidian 2= $(\text { flight service }- \text { taxi service })^{2}$

\subsection{Why Gravitational Search Algorithm Used?}

Genetic Algorithm is proposed as a search algorithm and has proven to be powerful in rapidly discovering good solutions for some difficult problems, especially when the search space is large, complex and poorly understood. But GSA calculates the overall force in the agents. In GSA, fitness value is important around the search space. In GSA fitness is reversely proportional to the distance between solutions. In the GSA, By increasing the number of repetitions of the algorithm, fitness value increased. In the web service selection as time increases, fitness value improved percentage doesn't decrease.Compared to the Genetic algorithm,Gravitational Search algorithm is best optimization algorithm in the travel plan domain.

\section{EXPERIMENTAL RESULTS}

\subsection{Comparing the Fitness Functions}

In this paper we compared the fitness functions of Genetic algorithm and Gravitational Search algorithm. Genetic algorithm Calculates the fitness values of whole iteration. So the time consumption is more. But, in GSA, choose fitness as random iteration of web service for calculate the fitness value. So the time consumption much lower compared to Genetic.

Table 1 Comparison between GA and GSA

\begin{tabular}{|c|c|c|}
\hline Iteration & \multicolumn{2}{|c|}{ Latency } \\
\hline \# & $\begin{array}{c}\text { Genetic } \\
\text { Algorithm }(\mathrm{ms})\end{array}$ & $\begin{array}{c}\text { Gravitational } \\
\text { Search } \\
\text { Algorithm }(\mathrm{ms})\end{array}$ \\
\hline 01 & 564 & 156 \\
\hline 02 & 609 & 109 \\
\hline
\end{tabular}

\begin{tabular}{|c|c|}
\hline 03 & 312 \\
\hline 04 & 234 \\
\hline 05 & 354 \\
\hline
\end{tabular}

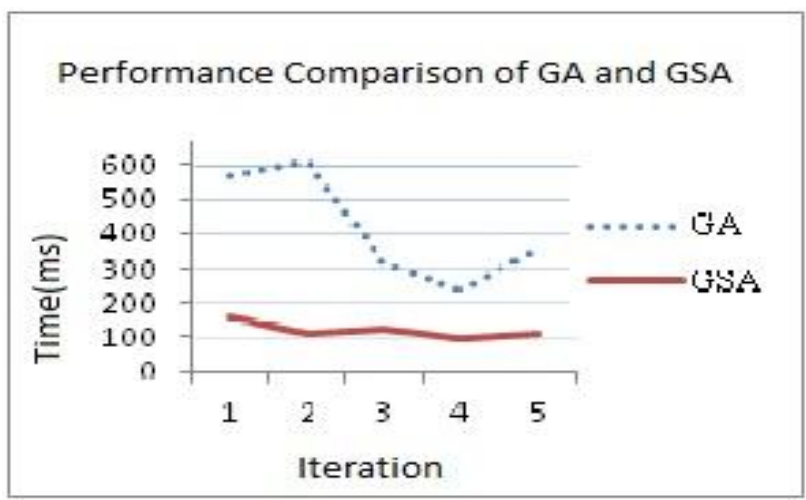

Fig. 3 Comparison of GA vs GSA

\subsection{Comparing GA with GSA}

The comparison results shown in Fig.3.For the particular time t, Gravitational Search algorithm performs better than Genetic algorithm. Gravitational Search algorithm solving the NP-hard problem like web service selection better than Genetic algorithm (Fig.4). This proves all the particle be likely to converge to the best solution quickly compared to Genetic algorithm. This is represented in the figure.(Refer Table 1)

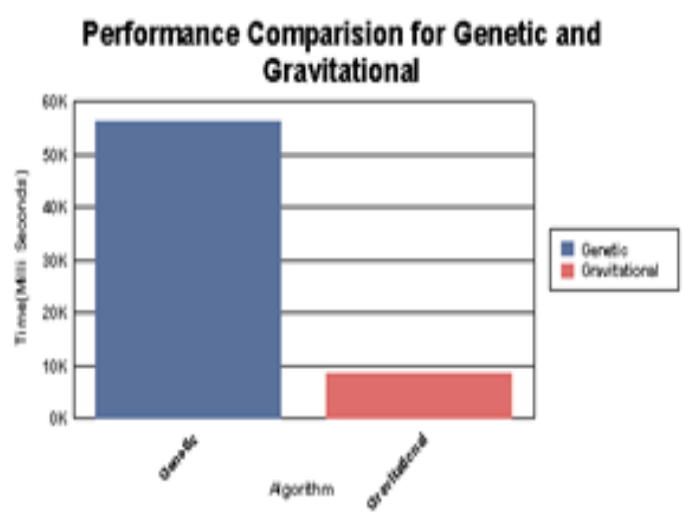

Fig.4 Bar Chart of Comparison

\section{CONCLUSIONS}

With the increasing number of web services, it is important to solve the problem of service selection to cater users need as a composite Web service. Since the existing service selection algorithms have defects such as inefficient, non-global optimization. The proposed Gravitational Search Algorithm based on user preference in the Web Service Selection is obtaining better results. The future work may be achieve towards incorporations other evolutionary Computing Algorithm the same. 


\section{REFERENCES}

[1] B. Zibanezhad, K. Zamanifar, N. Nematbakhsh, F. Mardukhi "An Approach for Web Services Composition Based on QoS and Gravitational Search Algorithm".

[2] Xue-Long Wang, B., Z. Jinq and H.Z. Yanq, 2011. "Service selection constraint model and optimization alogrithm for web service composition". MARCH2011

[3] Xi Chen, Xudong Liu, Zicheng Huang, and Hailong Sun "Web Services Composition and Optimizing Algorithm Based on QoS".

[4] Chen, X., X. Liu, Z. Huang and H. Sun, 2010. "RegionKNN: A scalable hybrid collaborative filtering algorithm for personalized web service recommendation (ICWS)". Proceeding of the IEEE International Conference on Web Services, Issue Date: 5-10 July 2010

[5] E. Rashedia, H. Nezamabadi-pour, and S. Saryazdi, "GSA: A Gravitational Search Algorithm", Elsevier, New York, NY, USA, June 2009, pp. 2232-2248.

[6] Goldberg, D. E. 1989. "Genetic algorithms in search, optimization and machine learning". Addison-Wesley Longman Publishing Co., Inc.,

[7] Esmat Rashedi, Hossien Nezamabadi-pour, Saeid Saryazdi, Malihe M. Farsangi. "Allocation of Static Var Compensator Using Gravitational Search Algorithm".

[8] R.Manoharan, A.Archana and Siddhika Cowlagi "Hybrid Web Services Ranking Algorithm". IJCSI, Vol 8, Issue 3, No.2, May 2011.

[9] Demian Antony D'Mello, Ananthanaranyana. “A Review of Quality of Service (QoS) Driven Dynamic Web Service Selection Techniques" ICIIS 2010, Jul 29-Aug 01,2010, India.

[10] B Prashanth and Y Narahari "Efficient Algorithm for Combinatorial Auctions with Volume Discounts Arising in Web Service Composition".

[11] Al-Masri E., Mahmoud Q. H., "Discovering the best web service", (poster) 16th Intl. Conference on World Wide Web (WWW), 2007, pp. 1257-1258. (For QWS Dataset Version 1.0 or QWS Dataset Version 2.0).

[12] Chengwen Zhang., "Adaptive Genetic Algorithm for QoS-aware Service Selection". 2011 Workshops of International Conference on Advanced Information Networking and Application.
[13] Yves Vanrompay, Peter Rigole, Yolande Berbers., "Genetic Algorithm-Based Optimization of Service Composition and Deployment".

[14] Dmytro Zhovtobryukh., “A Petri Net-based Approach for Automated Goal-Driven Web Service Composition". Simulation 2007; 83; 33.

[15] Canfora G., Penta M., Di,Esposito R., and Villani M., "A lightweight approach for QoS-aware service composition", In Proc. of the 2nd Intl. Conference on Service Oriented Computing, New York, USA. 2004.

[16] W3C Working Group. Web services architecture http://www.w3.org/.

[17] Zhang Liang-Jie, Li Bing, and Chao Tian st al., "On demand Web services-based business process composition", In Proc. of the IEEE Intl. Conference on System, Man, and Cybernetics, Washington, USA, 2003.

[18] Wei-Hua Ai, Yun-Xian Huang, Hui Zhang, and Ning Zhou, "Web Services Composition and Optimizing Algorithm Based on QoS", 4th Intl. Conference on Wireless Communications, Networking and Mobile Computing, 2008. WiCOM '08, Oct. 2008.

[19] Ming Chen, Zhen-wu Wang, “An Approach for Web Services Composition Based on QoS and Discrete Particle Swarm Optimization", ACIS Intl. Conference on Software Engineering, AI, Networking, and Parallel/Distributed Computing, 2007, vol. 2, pp. 37 4.

[20] Demian Antony D’Mello, V.S.Ananthanaranyana., “A Tree Structure for Web Service Composition”.@2009.

[21] Joyce El Haddad, Maude Manouvrier, and Marta Rukoz, (2010) "TQoS: Transactional and QoS-Aware Selection Algorithm for Automatic Web Service Composition", IEEE Transactions on Services Computing, Vol. 3, No. 1.

[22] Martine De Cock, Sam Chung, Omar Haffez, (2007) "Selection of Web Services with Imprecise QoS Constraints", Proceedings of the IEEE/WIC/ACM International Conference on Web Intelligence.

[23] Su Myeon Kim, Marcel-Catalin Rosu., "A Survey of Public Web Services". www 2004, May 17-22, 2004, New York, USA. 